ECCOMAS

\section{Proceedia}

COMPDYN 2021

$8^{\text {th }}$ ECCOMAS Thematic Conference on Computational Methods in Structural Dynamics and Earthquake Engineering

M. Papadrakakis, M. Fragiadakis (eds.) Streamed from Athens, Greece, 28 - 30 June 2021

\title{
PREDICTING THE SHEAR CAPACITY OF REINFORCED CONCRETE SLENDER BEAMS WITHOUT STIRRUPS BY APPLYING ARTIFICIAL INTELLIGENCE ALGORITHMS
}

\author{
Zelda Spijkerman ${ }^{1}$, Nikolaos Bakas², George Markou' ${ }^{1}$, Manolis Papadrakakis ${ }^{3}$ \\ ${ }^{1}$ Department of Civil Engineering, University of Pretoria, South Africa \\ e-mail: u17056757@tuks.co.za; george.markou@up.ac.za \\ ${ }^{2}$ Scientific Coordinator, Computation-based Science and Technology Research Center, The Cyprus \\ Institute, Nicosia, Cyprus \\ e-mail:n.bakas@cyi.ac.cy \\ ${ }^{3}$ Institute of Structural Analysis \& Seismic Research, National Technical University of Athens, 9 \\ Iroon Polytechniou Str., Zografou Campus, GR-15780 Athens, Greece \\ e-mail:mpapadra@central.ntua.gr
}

\begin{abstract}
This paper focusses on the ongoing discussion of developing a single relationship that can accurately predict the shear capacity of slender, reinforced concrete $(R C)$ beams without stirrups. To date, the main approach used to predict the shear capacity of $R C$ beams, has been based on the derivation of a formula from experimental data. In this study, the approach uses the development of RC FEM models without stirrups, where the beam width is larger or equal to the section height and tested under three-point bending. The models were created and analysed by using Reconan FEA software, where the obtained results from the nonlinear analyses were used to construct a large database of 10,000 beams with varying material and geometric properties. Artificial Intelligence (AI) training was performed by using machine learning algorithms on the numerically generated database to develop predictive models and to develop an improved formula for predicting the shear capacity of $R C$ beams without stirrups. The proposed predictive formula was validated against an available ACI database of $R C$ beams that were assembled by using experimentally tested, physical beams without stirrups. The predictive formula was also compared with the design code formulae proposed by ACI 318-19 and Eurocode 2. According to the numerical findings of this research work, the proposed formula outperformed both design formulae demonstrating significant potential in replacing the current design approach.
\end{abstract}

Keywords: Shear Strength Prediction, Artificial Intelligence Algorithms, Design Formulae, Finite Element Method, Reinforced Concrete. 


\section{INTRODUCTION}

Shear failure of Reinforced Concrete (RC) beams takes place due to insufficient shear resistance in the web of the beams. It is important to sufficiently understand the shear capacity of beams in civil engineering applications to avoid catastrophic, brittle failures that occur suddenly, with little to no warning.

The behaviour of RC in shear has been a topic of discussion for numerous years, but the challenge of accurately predicting the shear capacity of $\mathrm{RC}$ beams remains an unresolved problem. The main approach so far has been to derive formulae using physical experiments and extrapolating the relevant values. This method is unfavourable, as a major disadvantage is insufficient available experimental data. It is impractical and infeasible to conduct enough physical experiments to cover all possible beam geometries, concrete and steel reinforcement properties, load cases and boundary conditions. The existing formulae are based on a limited number of physical experiments and do not consider other cases, such as the use of deep beams experiencing arc action, or different material types of reinforcement rebars. Therefore, the use of the current design formulae is in need of improvement and expansion.

With the rise of Artificial Intelligence (AI) and Machine Learning (ML) in the past 20 years, researchers and engineers have explored the use of ML algorithms on experimental data sets to predict the shear capacity of beams more accurately. To an extent, this method improved the predictive abilities for certain geometries of $\mathrm{RC}$ beams, but the restriction of available experimental data sets still limits the prediction capabilities. These studies were also numerically constrained and the accuracy of predicting the shear capacity of beams is unsatisfactory.

In this research paper, the approach originally introduced by Bakas et al. [1] is ex-tended. Instead of using the results obtained from limited experimental data sets, this newly developed procedure focusses on generating a large numerical database by using Finite Element Method (FEM) software and then using these models to train ML algorithms in order to predict the shear capacity of slender RC beams with no shear reinforcement. The proposed new formula will therefore solely be based on 3D nonlinear Finite Element Analysis (FEA). It is important to note that no physical experiments were used to generate or train the developed formulae. The physical experiments were used for validation purposes only.

This paper aims to more accurately predict the shear capacity of RC beams without stirrups that have smaller geometries, when compared to existing design formulae.

\section{NUMERICAL INVESTIGATION}

A numerical procedure was developed for a database of twenty different geometrical RC beams. The characteristics for each of these beams were then increased by a random generation of 500 material property combinations to produce a database containing 10,000 unique beams. The 3D approach presented in [2-4], was used in the modeling and nonlinear analysis of the beams.

\subsection{Development of Numerical Campaign}

The simply supported, slender, RC beams that were generated for the purpose of this research project used 20-noded isoparametric hexahedral finite elements to model the concrete and the steel plates that act at the supports, as well as 2-noded rod elements for the steel reinforcement bars.

Fig. 1 depicts how the typical generated model looked like. The simply supported, slender $\mathrm{RC}$ beam had the smallest mesh of all the constructed beams and spans 1,500 mm. The beam has a $300 \times 150 \mathrm{~mm}$ rectangular cross-section that was discretized by using hexahedral elements that are $150 \times 150 \times 75 \mathrm{~mm}$ in the $\mathrm{x}, \mathrm{y}$ and $\mathrm{z}$ directions, respectively. 
A total of 76 hexahedral elements were used of which 4 were for the steel base plates at the supports, 48 for the concrete hexahedral elements and 24 for the steel reinforcement bars. In Fig. 2 the tensile longitudinal reinforcement bars at the bottom of the beam are embedded rod elements and the applied force at midspan is also visible.

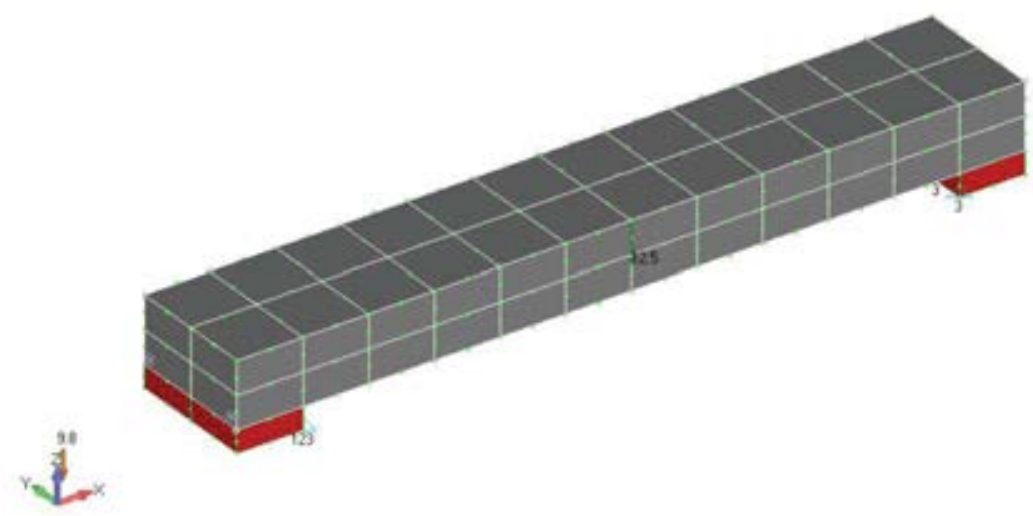

Figure 1. Hexahedral element mesh of a simply supported slender RC beam that has a net span of 1,500 mm and a $300 \times 150 \mathrm{~mm}$ cross section.

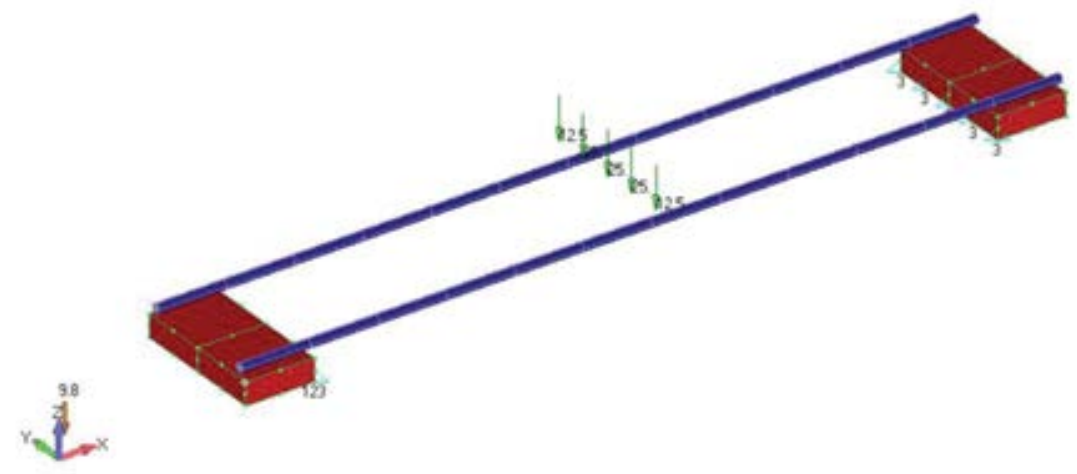

Figure 2. Embedded rod element mesh of a simply supported RC beam that has a net span of 1,500 $\mathrm{mm}$ and a 300x150 mm cross section with loads shown.

The largest mesh that was developed for this research project consisted of 450 hexahedral elements, where 10 elements were used for the steel support plates, 400 for the hexahedral elements and 40 for the steel rebars. The beam has a geometry of 750x350 mm and spans 2,700 $\mathrm{mm}$. The concrete cover for all the beams depended on the height of the section. Beams with a height smaller or equal to $250 \mathrm{~mm}$ had a concrete cover of $20 \mathrm{~mm}$ and beams with a height larger than $250 \mathrm{~mm}$ were modelled to have a concrete cover of $30 \mathrm{~mm}$.

\begin{tabular}{lll}
\hline Parameter & Minimum & $\begin{array}{l}\text { Maxi- } \\
\text { mum }\end{array}$ \\
\hline $\mathrm{L}(\mathrm{mm})$ & 1,500 & 2,700 \\
$\mathrm{~b}(\mathrm{~mm})$ & 300 & 750 \\
$\mathrm{~h}(\mathrm{~mm})$ & 150 & 350 \\
$\mathrm{~L} / \mathrm{h}$ & 6 & 10.8 \\
$\mathrm{~h} / \mathrm{b}$ & 0.33 & 0.83 \\
\hline
\end{tabular}

Table 1: Minimum and maximum beam geometries used in generating the 20 new models.

Twenty new beam geometries were constructed to generate the database. Only beam geometries where the width is greater or equal to the height were constructed. Table 1 shows a 
summary of the constructed models. The first model was generated with the minimum span, height and width values from Table 1 as can be seen in Fig. 1 and the subsequent models were generated by modifying the first model to get the needed geometries and spans. The span values start at a minimum of $1,500 \mathrm{~mm}$ and increase with $300 \mathrm{~mm}$ up until $2700 \mathrm{~mm}$. Span over section height $(\mathrm{L} / \mathrm{h})$ and the section height over the width $(\mathrm{h} / \mathrm{b})$ is also given in Table 1 with minimum and maximum values of 6 and 0.33 as well as 10.8 and 0.83 , respectively.

\subsection{Analysing Multiple Finite Element Beams}

To generate a database with sufficient input files, a certain level of automation is needed. Without automation, the required time to generate and analyse the input data would be a major disadvantage. The manual effort required to create the data would reduce the possible scope and negatively impact the resulting benefit of the research project. It was thus important to use automation code for data creation and entry. To minimise the time spent on producing and analysing the various FE models it was advantageous to use the previously developed automation tools from [1], Reconan Multirun v1.00.

A random generation of material properties combinations, consisting of $f_{c}(k P a), E_{c}(k P a), f_{t}$, $\beta, E_{\mathrm{s}}(\mathrm{kPa}), \mathrm{f}_{\mathrm{y}}(\mathrm{kPa})$ and $\mathrm{A}_{\mathrm{s}}\left(\mathrm{m}^{2}\right)$, was performed and used to generate the corresponding input file for each individual model. Each model's parameter input file included the node ID that had to be monitored, the direction in which the deflection was monitored, maximum deformation, the beam span as well as the section geometry with section height and section width. In all the generated models the node that was identified for monitoring was chosen as the nodal point at the centre of the total span, at the bottom of the beam's height and in the middle of the width.

The Reconan Multirun software was developed to modify each of the 20 model meshes that were generated to have the required material property assumptions and reinforcement ratios as described herein. The Reconan FEA [5], which was also algorithmically modified to read and analyse these FE input files to return the maximum capacity of the beam as well as the maximum deflection. It must be noted that, Reconan Multirun can also read the generated output files with the purpose of continuing the analyses if it was interrupted. This is a useful feature as unplanned power failures or system interruptions, such as operating system up-dates, would otherwise result in substantial delays and rework.

For each of the 20 models constructed as described in section 2.1, 500 different material properties and reinforcement ratio combinations were used, thus resulting in 10,000 analysis results that were used in training the AI algorithm.

The personal computer (PC) used for this research project had a 12-core i7-8700K CPU @ 3.70 GHz and 64.0 GB RAM and had the capability to run 10 Multirun analyses at the same time. As the model meshes became larger, with a larger span and geometry, the computational time to run the analyses increased. The entire analyses of all 10,000 models completed in roughly 36 hours, highlighting the computational efficiency of Reconan FEA [5] in performing nonlinear analysis using 3D detail models.

\section{DEVELOPMENT OF THE PROPOSED FORMULA}

For the second phase, the data was prepared by selecting, pre-processing and transforming the data to apply ML algorithms. The AI training proceeded by extracting the material features and predicting the shear strength of the beams. ML algorithms learn from data, so it was crucial that the right, quality data is fed into the system. The results achieved by the ML algorithms are directly related to the quality and volume of data used during the training stage.

Data selection for this project entailed generating a database that consisted of ten independent variables, which were split into two groups. The first group involved the variables that were 
used during the meshing of the models. These variables included the net span, $\mathrm{L}(\mathrm{mm})$, effective depth, $d(\mathrm{~mm})$ and width, $b(\mathrm{~mm})$. The second group of variables were strength-and material related properties and consisted of: uniaxial compressive strength of a cylindrical specimen $\left(f_{c}\right.$ in $\mathrm{MPa}$ ), concrete tensile strength ratio $\left(\mathrm{f}_{\mathrm{t}}\right)$, steel yielding stress $\left(\mathrm{f}_{\mathrm{y}}\right.$ in $\left.\mathrm{MPa}\right)$, Young's modulus of steel $\left(\mathrm{E}_{\mathrm{s}}\right.$ in $\left.\mathrm{MPa}\right)$, Young's modulus of concrete $\left(\mathrm{E}_{\mathrm{c}}\right.$ in $\left.\mathrm{MPa}\right)$, remaining shear capacity strength factor $(\beta)$ and the tensile longitudinal reinforcement ratio $(\rho)$.

The above-mentioned independent variables are presented in Table 2 with the statistical characteristics and minimum and maximum values of the database.

\begin{tabular}{lcccccccccc}
\hline & $\mathrm{L}$ & $\mathrm{d}$ & $\mathrm{b}$ & $\mathrm{f}_{\mathrm{c}}$ & $\mathrm{f}_{\mathrm{t}}$ & $\mathrm{f}_{\mathrm{y}}$ & $\mathrm{E}_{\mathrm{s}}$ & $\mathrm{E}_{\mathrm{c}}$ & $\beta$ & $\rho$ \\
\hline$\overline{\mathrm{X}}$ & 2100 & 242 & 537.5 & 41 & 0.061 & 500.8 & 200045.8 & 30024.9 & 0.034 & 0.0110 \\
$\sigma$ & 424.3 & 59.5 & 170.2 & 11.3 & 0.023 & 58.5 & 5602.2 & 2952.9 & 0.008 & 0.0053 \\
Median & 2100 & 230 & 500 & 40.9 & 0.061 & 499.2 & 200070.1 & 30072.1 & 0.034 & 0.0111 \\
Minimum & 1500 & 130 & 300 & 20.1 & 0.02 & 401.2 & 190000 & 25000 & 0.02 & 0.0016 \\
Maximum & 2700 & 320 & 750 & 59.9 & 0.1 & 598.5 & 210000 & 35000 & 0.05 & 0.0199 \\
$\mathrm{c}_{\mathrm{v}}$ & 0.202 & 0.246 & 0.317 & 0.277 & 0.377 & 0.1117 & 0.028 & 0.098 & 0.247 & 0.479 \\
\hline
\end{tabular}

Table 2: Statistical values of independent variables.

\subsection{Model Training}

The training process of any ML algorithm model can be split into 3 major phases: Pre-processing, Feature Selection and Extraction and Prediction.

Pre-processing of the assembled dataset was conducted by normalising the data by dividing each variable in the set by the standard deviation. The normalisation of the dataset was required because the features of the set have different ranges and consists of some outliers. The ranges were however not distorted, and the values of the different variables were changed to fit to a shared scale. Normalisation reduces duplicates in the database and increases the validation accuracy of the model. It also allows the optimization of the ML model to be more robust, as the convergence rate of the gradient descent is reduced.

Feature (or variable) selection then uses a process of dimensionality reduction to decrease the initial set of normalised data to more manageable groups. It greatly improves the ML algorithm's performance and reduces the computational power needed to perform the training. Following on from the feature selection is feature extraction. When a number of features in a dataset become similar or too large, the ML model can suffer from overfitting where the model fits the data too well and is no longer able to fit additional data or predict future observations in a consistent way. Feature extraction is the method for combining certain variables into features, reducing the number of values in the dataset without losing an accurate representation of the original data. The relevance and optimisation of the ML model is also considered during feature selection and extraction.

The algorithm chosen for the prediction phase of the ML and AI process is the NLR (nonlinear regression) model. Regression models are used to predict unknown values which are gathered from continuous (regression) variables and the NLR model is consequently beneficial to the research project and produces a closed-form solution. The prediction developed by the NLR model is presented as a formula in section 3.2 of this manuscript.

It is important to note that $85 \%$ of the generated database was used to train the algorithm, and the remaining $15 \%$ used for testing purposes. The test data proved that the prediction model that was created is accurate. The robustness of the model will be discussed in the next section. In addition, a set of physical RC beams was used for validation purposes, after the testing was performed. This step, which is considered to be the most important, was performed so as to 
check whether the proposed formula is able to predict the shear capacity of real beams that were experientially tested.

\subsection{Proposed Predictive Equation}

The formula developed during this research project, used to predict the ultimate characteristic shear strength $V_{c}$, utilized the NLR ML algorithm and was mainly dependent on $L, d, b, f_{c}$ and $\rho$. Formulae using 10, 100 and 500 terms were generated and investigated for robustness and accuracy. The higher order NLR that was implemented in this research project consists of a combination of variables that were created up to $3^{\text {rd }}$ order. The methodology uses the RFalgorithm (random forests) and the analyses was run on the Julia programming language as written by Dimopoulos and Bakas [6].

During the comparison of the three initially developed formulae, the mean absolute error (MAE) of the ten-term formula was 5.1\% higher than the 100 and 500 term formulae, and the MAE for the 100 and 500 term formulae was the same and equal to $6.89 \%$.

The proposed formula to calculate $\mathrm{V}_{\mathrm{c}}$, was obtained as output from the ML algorithm. The formula with ten-terms and the variables $L, d, b, f_{c}, f_{t}, \rho, \beta$, Es and corresponding weights are given in Eq. 1. The 10, 100 and 500 term formulae have been validated against the experimental data to determine the most accurate formula.

$$
\begin{array}{rl}
V_{c}=0.253939 & * \rho * d^{2}-0.0571682 * d * L * \rho+0.0967954 * \rho * b * d \\
& +0.0000000193334 * E_{S} * f_{c} * d-0.000000234107 * f_{c} * L^{2}+0.003624 \\
& * \rho * L^{2}-0.00809799 * \rho * L * b+0.00000538644 * f_{c} * b * d+343643 \\
& * \rho * f_{t} * \beta-387.761 * \rho^{2} * d+0.950862
\end{array}
$$

\section{VALIDATION OF PROPOSED EQUATION THROUGH EXPERIMENTAL DATA}

The validation of the predictive model was conducted using 36 experimentally tested beams that were taken from the ACI database [7] of shear tests on slender reinforced concrete beams without stirrups. The 36 beams were specifically selected to fall within the range of the 20 different beam geometries that were developed for the needs of this research project. The material properties were also within the boundaries of the generated database of the 10,000 models. Any experimental results from the ACI database that had incomplete data, were removed and not included in the validation beams.

It is necessary to mention that the ACI experimental database did not include values for $f_{t}$ and $\beta$. To include these parameters, the average value of $f_{t}$ of the training set was taken. $\beta$ was chosen as a random percentage of less than $5 \%$. This selection was made to optimise the results from the predictive model and to enhance the performance and accuracy. It is also important to note that all the validation beams were out-of-sample cases, given that the values of material properties and geometrical features differed in comparison to the values adopted for the generation of the 10,000 numerical results.

An additional observation related to the validation data is that, from the 36 beams in the ACI database [7] of experimentally tested beams, there were instances where beams with exactly the same beam geometry, reinforcement ratio and material properties resulted in different ultimate shear capacities. This finding highlights the level of uncertainty when discussing the physically tested beams and confirms that experimental results typically include noise because of uncontrolled factors during the testing phase.

It is noteworthy that the predictive model was successfully constructed without the use of any physical experimental data. By removing the dependency on physical beam data, it was 
possible to work with a much larger data set and avoid the inconsistencies inherent in physically collected data. The predictive formula, and the resulting accuracy, is purely based on the results obtained from the FEA. The experimental data used in this section was for validation purposes only.

Two industry-standard formulae were compared with the new proposed model, namely the ACI 318-19 [8] and Eurocode 2 [9]. The data in the experimental data set, with 36 RC beams without stirrups, were used to calculate a predicted shear capacity strength using the industrystandard formulae. The same data were used with the new model and the results were compared against the standard design formulae.

The ACI formula is expressed in imperial units so the data were first converted to the correct metric unit before calculations were made. Eq. 2 is the newly developed ACI 318-19 predictive formula that was published in 2019 and it is more similar to the design code suggested by Eurocode 2 [9]. In the case of this research project, no axial force was present, and $N u$ is irrelevant $(N u=0)$. In the ACI formula, the beam geometry and concrete strength plays an important role. The reinforcement ratio $(\rho)$ also has an impact on the shear capacity of the RC beam. The formula in Eurocode 2 (Eq. 3), dependents on the reinforcement ratio, strength of concrete and the beam geometry.

$$
\begin{gathered}
V_{c}=\left[8 \lambda_{s} \lambda\left(\rho_{w}\right)^{1 / 3} \sqrt{f_{c}^{\prime}}+\frac{N_{u}}{6 A_{g}}\right] b_{w} d \\
V_{R k, c}=C_{R k, c} k\left(100 \rho f_{c k}\right)^{1 / 3} b_{w} d \\
\text { with } k=1+\sqrt{\frac{200}{d}} \leq 2.0(\text { d in } m m) \text { and } \rho=\frac{A_{s l}}{b_{w} d}
\end{gathered}
$$

Both formulae were developed based on the results obtained from physically constructing and testing several beams, therefore, they are both semiempirical. The obtained results are graphically displayed in Fig. 3 to 5. In each figure, the physically tested, experimental results are displayed on the horizontal $\mathrm{x}$-axis and the shear capacity at which the beams were predicted to fail, using different formulae and models, is displayed on the vertical y-axis.

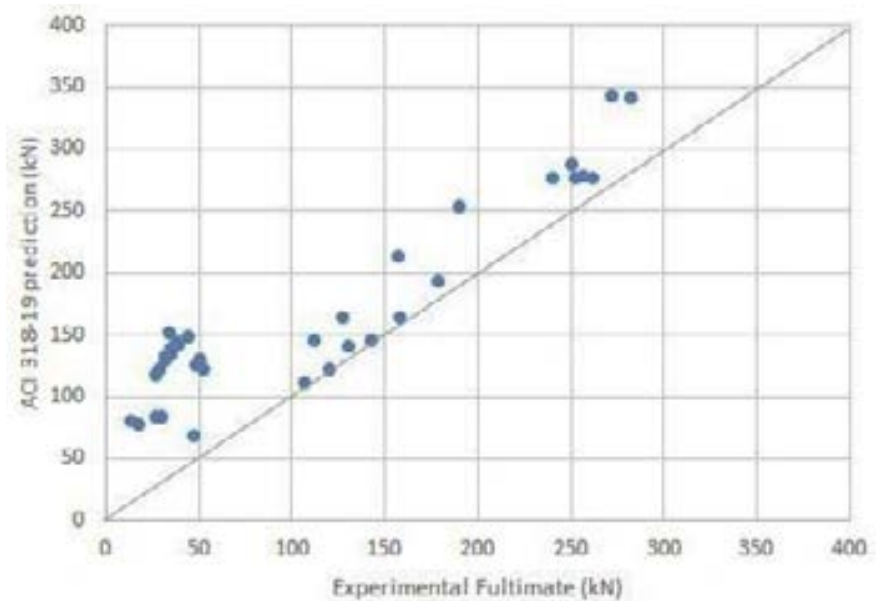

Figure 3. Experimental Shear Strength vs ACI 318-19 Prediction.

The influence of the longitudinal reinforcement ratio, $\rho$, impacts the outcome of the shear capacity, providing the ACI 318-19 formula with some accuracy. However, the ACI formula 
tends to over-predict the ultimate shear capacity of the beams, meaning that a much higher shear strength was calculated than the actual shear strength that the beam could resist.

The accuracy of the Eurocode 2 prediction of the ultimate shear strength of RC beams without stirrups is higher than the ACI formula, with a MAE of 1.1298, thus tt is considered more accurate than the 2019 version of the ACI code when implemented on the validation set of beams. From Fig. 4 it is seen that for experimental values of $V_{c}$ less than $50 \mathrm{kN}$, the prediction calculates a much larger value and is less accurate, whereas values of $\mathrm{V}_{\mathrm{c}}$ larger than $50 \mathrm{kN}$ are close to the predicted values.

Fig. 5 compares the experimental shear strength of the RC beams with the new, improved predictive ten-term formula developed by using NLR. When compared to Figs 3 and 4, and looking at the newly calculated MAE, the new ten-term formula is found to be more accurate and best predicts the shear capacity of the beams which fail at a higher ultimate force.

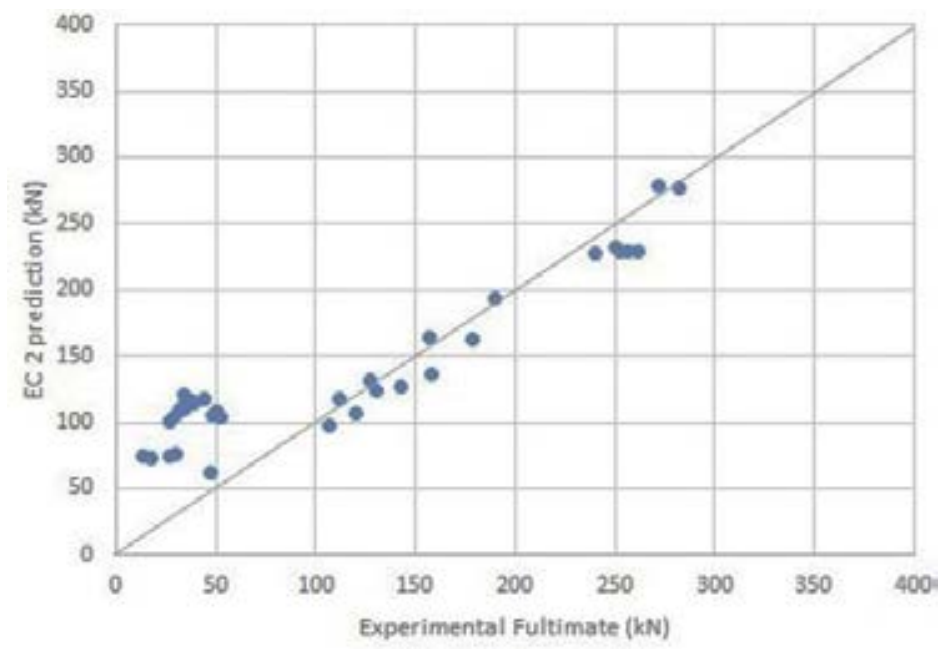

Figure 4. Experimental Shear Strength vs EC 2 Prediction.

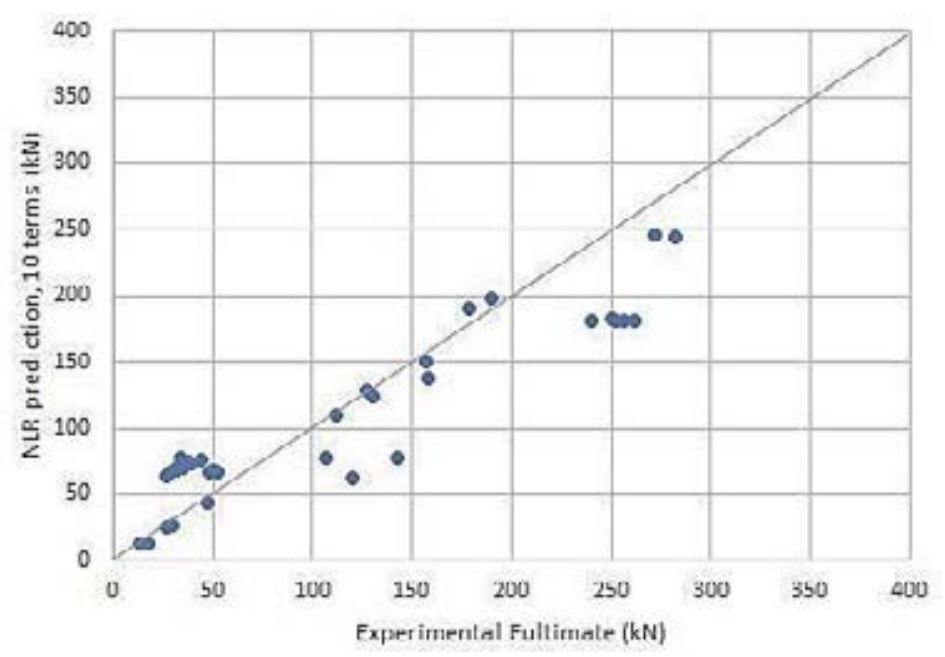

Figure 5: Experimental Shear Strength vs NLR prediction formula with ten-terms.

For the new formula the overall distribution of the datapoints is closer to the $\mathrm{x}=\mathrm{y}$ line, when compared to the ACI 318 and Eurocode 2 predictions. The proposed predictive formula results look visually similar to those presented in Figs 3 and 4, however, there is a closer and more accurate prediction when looking at ultimate forces smaller than $50 \mathrm{kN}$. 
The forces larger and equal to $50 \mathrm{kN}$ appear slightly less accurate when compared to the Eurocode formula predictions in Fig. 4. The proposed formula yields a more conservative numerical response to the problem by predicting forces smaller than the actual force at which the $\mathrm{RC}$ experimental beams fail.

The new 10-, 100- and 500-term formulae were compared by looking at their respective MAEs. The new 10-term formula was the most accurate by far, with a MAE of only 0.4703 . Thereafter the 100-term formula with a MAE of 0.6408 and the 500-term formula follows closely with a MAE value of 0.6616 . The MAEs of the different predictive models are shown in Table 3, with the lowest error resulting from the new 10-term NLR predictive model generated in this study.

Interestingly, although the model was trained on a dataset with different beams' width-height ratios, it exhibited high accuracy, compared to the Eurocode and ACI building codes. All three of the new NLR formulae composed in this study yield more accurate results than the existing industry-standard design formulae when used to predict the shear capacity of the 36 validation $\mathrm{RC}$ beams. The ten-term formula provides the best fit and has the lowest error. Although overfitting is present with both the 100- and 500-term formulae they also provide a lower MAE when compared to the existing industry-standard formulae.

\begin{tabular}{lll}
\hline Predictive Model & MAE $(\%)$ & Accuracy \\
\hline 10-term NLR & 47.03 & Best \\
100-term NLR & 64.08 & \\
500-term NLR & 66.16 & \\
Eurocode 2 & 112.98 & \\
ACI318-19 & 145.51 & Worst \\
\hline
\end{tabular}

Table 3: MAE of predictive models.

\section{CONCLUSIONS}

Non-linear regression algorithms were used to train and test a database that consisted of 10,000 shear strength results. Removing the requirement for physical beam data enabled the creation of a much larger data set, which made it possible to use Machine Learning techniques. The shear strength results from the physical experimental data were only used to validate the results obtained from the combination of the FEA and ML algorithms.

The proposed predictive formula with 10-terms for computing the shear capacity of slender $\mathrm{RC}$ beams without stirrups presented in this research project outperformed the existing available design code formulae (ACI 318-19 and Eurocode 2). The new formula was shown to be more accurate and better predicted the shear capacity of $\mathrm{RC}$ slender beams without shear reinforcement. FEA used in conjunction with advanced ML was shown to provide a reliable approach in developing a predictive formula for estimating the capacity of $\mathrm{RC}$ beams without stirrups.

The proposed predictive formula can be further improved by extending the current generated database with a larger variety of beam geometries, spans, and material properties that fall outside of the current range. Further research can be done on different design codes, such as the Canadian code, and formulae used around the world to compare the accuracy of currently used design formulae.

Furthermore, it is recommended to build on this research by looking at slender beams with shear reinforcement, beams with compressive longitudinal reinforcement bars, deep beams, slabs and RC beams with fibre reinforced polymer rebar as well as RC beams with fibres. Additionally, columns, shear walls and joints should be investigated under monotonic and cyclic loading conditions. 


\section{REFERENCES}

[1] N. Bakas, G. Markou, D. Charmpis and K. Hadjiyiannakou, "Performance and scalability of deep learning models trained on a hybrid supercomputer: Application in the prediction of the shear strength of reinforced concrete slender beams without stirrups", COMPDYN 2021, 27-30 June 2021, Streamed from Athens, Greece.

[2] G. Markou, N.P. Bakas, Computationally efficient 3D finite element modelling of RC structures, Computers and Concrete, Vol 12, No 4, May, pp 443-447, 2013.

[3] G. Markou and F. Genco, Seismic Assessment of Small Modular Reactors: NuScale Case Study for the 8.8 Mw Earthquake in Chile, Nuclear Engineering and Design, 342(2019), pp. 176-204, 2019.

[4] C. Mourlas, G. Markou and M. Papadrakakis, Accurate and Computationally Efficient Nonlinear Static and Dynamic Analysis of Reinforced Concrete Structures Considering Damage Factors, Engineering Structures, 178 (2019), pp. 258-285, 2019.

[5] Reconan FEA v2.00, User's Manual, 2020. https://www.researchgate.net/publication/342361609 ReConAn v200 Finite Element Analysis Software User's Manual

[6] T. Dimpoulos and N. Bakas, Sensitivity analysis of machine learning models for the mass appraisal of real estate. Case study of residential units in Nicosia, Cyprus, Remote Sensing, vol. 11, no. 24, p. 3047, 2019.

[7] K.-H. Reineck, E.C. Bentz, B. Fitik, D.A. Kuchma and O. Bayrak, ACI-DAFSTB database of shear tests on slender reinforced concrete beams without stirrups, ACI Structural Journal, 110 (5), 2013.

[8] A.C. Institute, Building Code Requirements for Structural Concrete (ACI 318-19): Commentary on Building Code Requirements for Structural Concrete (ACI 318R-19): an ACI Report, American Concrete Institute. ACI, 2019.

[9] EN1992-1-1, Eurocode 2 (2004), Design of concrete structures. 